\title{
Coinfection Organizes Epidemiological Networks of Viruses and Hosts and Reveals Hubs of Transmission
}

\author{
Michael McLeish, ${ }^{\dagger}$ Soledad Sacristán, Aurora Fraile, and Fernando García-Arenal \\ Centro de Biotecnología y Genómica de Plantas Universidad Politécnica de Madrid (UPM)-Instituto Nacional de Investigación y Tecnología \\ Agraria y Alimentaria (INIA) and Escuela Técnica Superior de Ingeniería Agronómica, Alimentaria y de Biosistemas, Universidad \\ Politécnica de Madrid, Madrid 28223, Spain \\ Accepted for publication 10 December 2018.
}

\begin{abstract}
Multiple virus infections affect the competence of host plants to transmit disease. The effects of coinfection on transmission are expected to produce ecologically complex pathogen and host-pathogen interactions. However, the prediction of disease risk will rely on untangling nonrandom from random patterns of infection to identify underlying processes that drive these interactions. Are the spatial distributions of infections in complex multispecies systems random or not? For the first time, we use an empirical evaluation of this basic but nontrivial question to test the hypothesis that coinfection contributes to (i) nonrandom ecological interactions between hosts and viruses and (ii) structuring infection distributions. We use a novel approach that decomposed the ecological interactions of 11 generalist viruses in 47 host species in four habitats of an agroecosystem into single-infection and coinfection "modes." Then, we relate ecological structuring in infection networks to the distribution of infection using generalized regression models. The

network analyses of coinfection showed that virus-host interactions occurred more often than expected at random in one of the four habitats, Edge. A pattern of specific interactions was shared between Edge and the ecosystem, indicating scale invariance. The regression modeling also showed that the plant community characteristics of Edge were unique in explaining infection distributions. The results showed that the spatial distribution of infection at the ecosystem level was not only a speciesspecific phenomenon but also, strongly structured by specific virus-virus and host-virus interactions. The evidence of scale invariance and the special role of Edge as a reservoir suggest that ecological interactions were less strongly structured by community differences among habitats than by wider-scale processes and traits underlying the interactions. Addressing whether reservoir communities significantly contribute to epidemiological processes at the ecosystem scale is a promising avenue for future research.
\end{abstract}

Plant pathogens that infect multiple species have great potential to interact with a diversity of other pathogens via coinfection (Woolhouse et al. 2001). Coinfection of an individual can produce synergistic to antagonistic interactions between pathogens that are important to transmission dynamics (Syller 2012). However, the relative importance of coinfection has been underestimated by the single host-single pathogen studies that tend to dominate the literature (Barrett et al. 2009; Woolhouse et al. 2002). Coinfection is expected to affect species interactions that drive the risk of disease in natural environments and add to the complexity of transmission (Telfer et al. 2010; Tollenaere et al. 2016). The complexity of ecological interactions will, therefore, add to the difficulty of identifying nonrandom spatial effects on the distribution of infection. The identification of nonrandom distribution patterns of infection is an important step for predicting transmission pathways in natural communities.

In multispecies systems, general factors that explain the distribution of infections are largely unknown, because it is unclear how environmental heterogeneity affects the susceptibility of hosts and their competence to transmit pathogens (Streicker et al. 2013). Environmental heterogeneity also has implications for the movement ecology of vector-borne pathogens (Ostfeld and Keesing 2000). Generally, transmission depends on ecological interactions among pathogens and the hosts that they infect (Gurarie and Seto 2008; Yang

†Corresponding author: M. McLeish; michael.mcleish@upm.es

Funding: This work was in part supported by grant BFU2015-60418-R from Plan Estatal de I+D+i, Spain (to F. García-Arenal).

*The $\boldsymbol{e}$-Xtra logo stands for "electronic extra" and indicates that one supplementary file, eleven supplementary figures, and six supplementary tables are published online.

The author(s) declare no conflict of interest.

(C) 2019 The American Phytopathological Society et al. 2015). Infection heterogeneity, where a small proportion of hosts supports a large proportion of pathogens, is a common observation in many systems (McLeish et al. 2017; Streicker et al. 2013; Susi et al. 2015) and implies nonrandom patterns of infection distributions in natural environments. Coinfection is also common (Lello et al. 2004; Malpica et al. 2006; Woolhouse et al. 2001), and it is hypothesized to affect virulence and pathogen accumulation, the generation and maintenance of genetic diversity, and ultimately, transmission (Tollenaere et al. 2016). Understanding how the distributions of host plants and coinfecting pathogens overlap or not is critical to uncovering mechanisms that drive transmission.

The distribution of plants in agricultural ecosystems is characterized by high variation in density over space in areas usually comprising crops, semiwild pastures, and various successional assemblies, such as fallow fields. Measures used to evaluate variation in these cover types, so-called compositional heterogeneity, include diversity parameters, such as richness and evenness (Fahrig et al. 2011). Strong compositional heterogeneity in agroecosystems affects transmission, because it influences the spatiotemporal mixing of plants, pathogens, and vectors, structuring pathogen distributions (Buhnerkempe et al. 2015; Malmstrom et al. 2006; Perkins et al. 2013; Power and Mitchell 2004; Streicker et al. 2013; Viana et al. 2014). For instance, in a multihost, multipathogen system, infection, transmission, and coinfection of parasitic fungal pathogens depended on functional groups of herbs, grasses, and legumes in communities of up to 60 species (Rottstock et al. 2014). Compositional heterogeneity among habitats of an agricultural ecosystem is, therefore, expected to generate nonrandom ecological interactions among viruses and host plants and drive transmission and infection heterogeneity. Furthermore, coinfection may also mediate infection heterogeneity, because transmission is an emergent property of within-host and between-host processes.

Network-based approaches are useful for identifying nonrandom associations present in biologically complex interactions (Ings et al. 
2009; Lewinsohn et al. 2006). For instance, bipartite networks have been used to infer the spread of infectious disease in heterogeneous environments (Craft et al. 2010; Leventhal et al. 2015). One of the major observations made from ecological interaction networks is the tendency for some species interactions to be compartmentalized, a quality known as modularity. Modularity is a measure of ecological interactions and the division of a network into compartments (Krause et al. 2003; Newman and Girvan 2004). Coinfection is expected to increase the ecological interactions of plants and pathogens at the individual and community levels and reveal important components of transmission dynamics.

Generalist plant viruses offer an excellent opportunity to investigate ecological interactions resulting from coinfection and identify components of the system that are essential to transmission. For example, Seabloom et al. (2010) related virus diversity to coinfection rates of barley and cereal yellow dwarf viruses in Bromus hordeaceus, Elymus glaucus, and Avena fatua. The study found that virus coinfection was conditional on plant community type, vector ecology, and environmental factors. In another study, Seabloom et al. (2009) used an empirical model to show that coinfection of the native grass E. glaucus by communities of luteoviruses and poleroviruses was associated with vector ecology more so than cross-protective immunity mechanisms within the host. Vector behavior changed according to the plant communities in which host species occurred. Coinfection might also add a degree of stochasticity to interactions in a plant-pathogen system. For example, at a comparatively small scale in a wild population of Cucurbita pepo, coinfection by Zucchini yellow mosaic virus (ZYMV) and Watermelon mosaic virus (WMV) resulted in differential plant fitness costs that were dependent on the type of virus-virus interaction (Salvaudon et al. 2013). If the spatial distribution of the type of coinfection is random, it implies that the distribution of plants with low/high fitness is also random.

The objective of this study was to identify and highlight the network of ecological interactions that was expected from coinfection and compare it with a baseline of single infections only. This approach makes it possible to determine whether the distribution of coinfections in the ecosystem generates wellconnected network architectures or not and if so, which species interactions were the strongest. Observations of 11 generalist viruses and the 47 plant species that they infected were related to four cover types (henceforth, "habitats") of an agricultural ecosystem. We measured infection heterogeneity using estimates of infection prevalence. Regression models were then used to evaluate the variance contribution to infection heterogeneity of either coinfection or diversity among the habitats (compositional heterogeneity). The network and regression approaches complement each other, because networks provide evidence of specieslevel interactions and their strength, whereas regression makes it possible to evaluate the distribution of infection at the community level. Specifically, we tested the following hypotheses that (i) coinfection contributes more than single infections to nonrandom associations and ecological interactions between hosts and viruses and that (ii) coinfection contributes to the distribution of pathogen prevalence among habitats. We found that the coinfection networks exhibited ecological interactions that resulted in strong (nonrandom) compartmentalization compared with the single-infection networks. The results of the two main analyses agree on the unique role of a particular habitat, Edge, in structuring infection in the ecosystem. Single infections were significantly related to habitat community parameters by contrast to coinfections, which were partly structured by compositional heterogeneity and largely structured by wider ecosystem-level processes.

\section{MATERIALS AND METHODS}

Sample collection. Sampling of leaf tissue took place from 2000 to 2002 in central Spain, with collections made in each season from two sites (i.e., four resamples $\times$ two sites per habitat) in each of four habitats: (i) the boundary immediately adjacent to cultivated fields (Edge), (ii) agricultural fields left fallow between crops at the time of sampling (Fallow), and (iii) abandoned sites largely undisturbed by current land use practices for $>2$ years interspersed among crops (Wasteland), and (iv) cultivated fields (Crop). Crops were absent during winter and spring and sampled in summer and autumn. Crops are separated by narrow borders of relatively permanent plant assemblages in Edge, which are left generally intact between seasonal cropping. Edge sites occasionally experienced low-level disturbance by either burning or partial plowing. Fallow communities assemble de novo at different times throughout the year owing to tillage cycles. Wasteland and Edge communities comprise successional assemblies that remained largely untouched by anthropogenic disturbance. The dimensions of each habitat vary but retain specific community characteristics, and they form a mosaic of patches across the ecosystem. The analysis of the sampling effort by rarefaction has been detailed in previous work (McLeish et al. 2017), and it indicated nearasymptotic curves for all habitats (Supplementary Fig. S1). The distinctions among the habitats and their community characteristics were also described. The study sites were dispersed over an area of $10 \mathrm{~km}^{2}$. The spatial relationships between Edge and Wasteland were constant, but the locations of Fallow and Crop varied every year. Leaf samples, regardless of showing symptoms, were collected from one plant at each of 25 fixed points along transects in $10 \times 10-\mathrm{m}$ quadrats at each site at each collection. Depending on the plant species, a number of leaf samples $(>5$ and $<20)$ from different parts of the individual were collected. Leaf samples were not collected if a plant was not present at a fixed point. Under systematic sampling, we assume that comparative frequencies of the observed infections represent realized interactions. The data are available from the Dryad Digital Repository (http://datadryad.org/).

Virus detection. Plant collections were analyzed using doubleantibody sandwich enzyme-linked immunosorbent assay (ELISA) (Clark and Adams 1977) to detect 11 single-stranded RNA (ssRNA) viruses (Supplementary Table S1): Alfalfa mosaic virus (AMV), Beet Western yellow virus (BWYV), Bean yellow mosaic virus (BYMV), Cucumber mosaic virus (CMV), Lettuce mosaic virus (LMV), Papaya ringspot virus (PRSV), Potato virus $Y$ (PVY), Tomato spotted wilt virus (TSWV), Turnip mosaic virus (TuMV), WMV, and ZYMV. These viruses are transmitted by aphid vectors in a nonpersistent manner, except BWYV, which is transmitted by aphids in a persistent, nonpropagative manner, and TSWV, which is transmitted by thrips in a persistent propagative way (Whitfield et al. 2015). A sample was considered as infected by a certain virus if the absorbance in the ELISA was greater than or equal to two times the negative controls (Supplementary File S1). The primary data included 16,148 observations that included 1,468 individual plants and the 11 viruses infecting them. Of the 1,468 records comprising 71 plant species, 395 individuals supported 608 infections of at least 1 of the 11 viruses, and 47 had at least one specimen infected by 1 virus.

Infection network analysis. Bipartite networks were constructed to quantify infections (links) between the 11 viruses and the 47 host plants (nodes). A complete list of taxon codes is given in Supplementary Table S2. Parallel links (more than one link joining a pair of nodes) were simplified and weighted in undirected networks. Network approaches assume a finite spatial-temporal boundary (i.e., the agroecosystem) with known taxonomic resolution, and observations were derived with a systematic procedure (Lewinsohn et al. 2006). Network links were defined as a single infection (links between a virus and individual host with one observed infection), coinfection (i.e., observations of individual hosts with more than one infection), or total infection (i.e., both single infections and coinfections) to compare modularity of each infection mode from each of the four habitats. Monopartite networks were generated by 
compressing the bipartite networks for convenient visualization of virus-only coinfection interactions. The links in the monopartite networks were weighted by the number of times that the corresponding relationship between two viruses was repeated. Because monopartite networks are less informative, we assessed the ecological interactions of each mode of infection using bipartite networks. Differences between the node degree cumulative frequency distributions (CFDs) $P(k)$ of the single-infection and coinfection networks were tested, and departures from expectations of the power law distribution were assessed using the KolmogorovSmirnov test. By contrast to random networks, many real networks have increasingly well-connected architectures that show departures from the expectations of a power law distribution (Barabási and Albert 1999). Modularity $(Q)$ was used to measure network compartmentalization and estimated using the Walktrap algorithm (Pons and Latapy 2006). The estimate of $Q$ was compared with a distribution of 1,000 randomized networks having the same number of nodes and links as in the observed network using a $t$ test. All network manipulations and analyses were conducted with the $\mathrm{R}(\mathrm{R}$ Core Team 2012) packages igraph (Csardi and Nepusz 2006) and NetIndices (Kones et al. 2009).

Modeling co-prevalence in the plant-pathogen system. We implemented generalized linear mixed models (GLMMs) using two designs to test the effect of compositional heterogeneity on infection heterogeneity measured as prevalence. Multiple competing models were used to statistically test the contribution of predictor variables to the variance structures of the models. We modified the measure of prevalence to explicitly account for the number of infections in an individual and called it co-prevalence. Co-prevalence is the sum of infections (of any of the 11 viruses, including single infections) detected in each individual sample of a given host species divided by the abundance of that species. Thus, co-prevalence depended on not only the mode of (single or multiple) infection but also, the number of different viruses coinfecting an individual (Supplementary Fig. S2). The probability distribution of the co-prevalence variable was modeled using kernel density estimation and compared with that of the distribution of the prevalence variable. The distribution of the co-prevalence variable was left skewed by comparison with that of prevalence. The leftskewed distribution could be corrected by using a log transformation. Therefore, an inverse gamma distribution was used to relate the fixed factors to co-prevalence, and a log link was used between the error terms of the random factor and predictors in the generalized regression models.

The first GLMM design enabled us to test temporal and spatial effects and the relative importance of virus or host species identity on co-prevalence while specifying a factor for infection mode used to distinguish singly from coinfected individuals as in the network analyses. These models included temporal factors for year and season, a spatial factor for habitat, plant community parameters for relative abundance (counts included infected and noninfected individuals of host species) and Tsallis diversity $\left(S_{q}\right)$ of host species, species factors for the host plant or virus, and infection mode (a binary factor for either singly infected individual or coinfected individual). Host plant diversity $S_{q}$ was estimated for each year/ season/habitat combination.

We used a second design ( $n=47$ host species subjects) to address whether coinfection was associated with habitat-specific fixed factors for relative host abundance. A random factor for coinfection level was introduced to assess differences in frequency of coinfection among the habitats. There were six separate levels of up to greater than six infections, with each host species as a subject, and the number of coinfections in each species summed at each level and treated as a factor. Model suitability was assessed with the Akaike information criterion (AIC) and residual deviance tests (RDTs). An RDT of 1.0 indicates that the model provides a good fit to the data. The models were implemented using the $\mathrm{R}$ package lme4 (Bates et al. 2015).

\section{RESULTS}

Network architecture and plant-virus communities. The results showed that, of a total of 608 links, $341(56 \%)$ represented coinfections, and $267(44 \%)$ represented single infections. The node degree $(k)$ cumulative frequency of each network indicated ecological interactions that exceeded expectations of a power law distribution visualized in Supplementary Figure S3. However, the null hypothesis that the single-infection and coinfection CFDs of the node degree belong to the same distribution was rejected (two-sample Kolmogorov-Smirnov, $D=0.622, P<0.0001$ ). The coinfection CFD showed a greater departure than the singleinfection CDF from the power law expectation. The greater the departure, the larger the proportion of highly linked nodes in the network (Supplementary Fig. S4). On average, there were significantly fewer weighted network links of single infection [paired two-tailed $t_{(10)}=-2.375, P$ value $=0.019$ ] than weighted links of virus coinfection (Fig. 1), and there were significantly fewer weighted network links of single infection than for coinfections [Welsh two-sample, two-tailed $t_{(44.68)}=-4.262, P<$ 0.001] among the host species (Supplementary Fig. S5). Both results indicated the dominance of coinfection in the ecosystem. The modularity $Q$ of the randomized sample mean was significantly less [one-sample $t_{(999)}=-5.486, P<0.0001$ ] than the observed estimate in the Edge coinfection network (Supplementary Table S3) and indicated that Edge had greater than expected modularity compared with the other habitats. The estimate for the number of modules in both the Edge and the Total coinfection networks was two. Together, these results showed that Edge had ecological interactions distinct from the other habitats but similar ecological structuring to all of the habitats combined at the ecosystem level.

The infection networks (Figs. 2, 3, and Supplementary Figs. S6 and S7) showed six main patterns. (i) AMV and CMV had the highest linkage specificity (the number of links between two nodes) of single-infection interactions. (ii) BWYV and TSWV had high-frequency interactions in the coinfection network. (iii) High linkage specificity by AMV and CMV differed in that they occurred either within or between modules, respectively. (iv) CMV tended to be linked to multiple hosts both within and between modules, whereas AMV tended to be linked within its module to highly connected multiple plant species. (v) Potyviruses, WMV, BYMV, LMV, ZYMV, TuMV, PRSV, and PVY, were

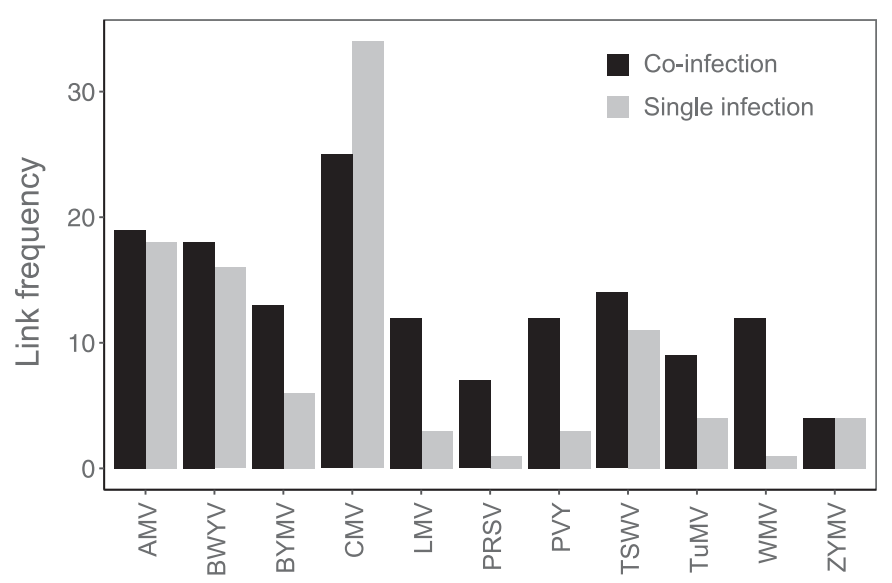

Fig. 1. Frequency distribution of links in weighted networks of the virus infection modes. Coinfections (black bars) and single infections (gray bars) are for all 47 host species. AMV, Alfalfa mosaic virus; BWYV, Beet Western yellow virus; BYMV, Bean yellow mosaic virus; CMV, Cucumber mosaic virus; LMV, Lettuce mosaic virus; PRSV, Papaya ringspot virus; PVY, Potato virus Y; TSWV, Tomato spotted wilt virus; TuMV, Turnip mosaic virus; WMV, Watermelon mosaic virus; and ZYMV, Zucchini yellow mosaic virus. 
found in the same module of the coinfection networks. (vi) The most frequent infections overall were by species (AMV, BWYV, CMV, and TSWV) of four virus genera. These viruses also had relatively wide host ranges (Supplementary Fig. S8) (based on infections detected in this study) on species that were relatively abundant (Supplementary Fig. S9). The largest group of viruses comprised CMV and the potyviruses, and they were connected within a module observed in both the Edge and ecosystem-level coinfection networks. Similarly, AMV, BWYV, and TSWV were members of the same module regardless of the network. Amaranthus sp. (H2), Convolvulus arvensis (H12), Diplotaxis erucoides (H16), and Fumaria sp. (H19), were often observed in the same module. D. erucoides (H16) and Lactuca serriola (H22) had strong ecological interactions between and within modules in most of the networks. By comparison, Medicago sativa (H27) had stronger within-module ecological interactions. Highly frequent coinfections in Edge species included $D$. erucoides (in a single individual: AMV, CMV, PVY, TSWV, and WMV), Trifolium pratense (in a single individual: AMV, BYMV, PRSV, TSWV, WMV, and ZYMV), and Xanthium strumarium (in a single individual: CMV, BYMV, LMV, PRSV, WMV, and ZYMV) as well as Amaranthus sp. (H2), C. arvensis (H12), and Fumaria sp. (H19). The same species also had frequent single infections in other habitats. Hosts, such as Amaranthus sp. (H2), D. erucoides (H16), L. serriola $(\mathrm{H} 22)$, and $M$. sativa $(\mathrm{H} 27)$, had high-frequency single infections and also supported high coinfection levels in other instances.

Coinfection and prevalence. The first series of GLMMs indicated that the factor for year had a relatively weak contribution to total variance of the co-prevalence response (Supplementary Table S4). We dropped year in subsequent models to optimize model simplicity and performance. A factor for season and habitat

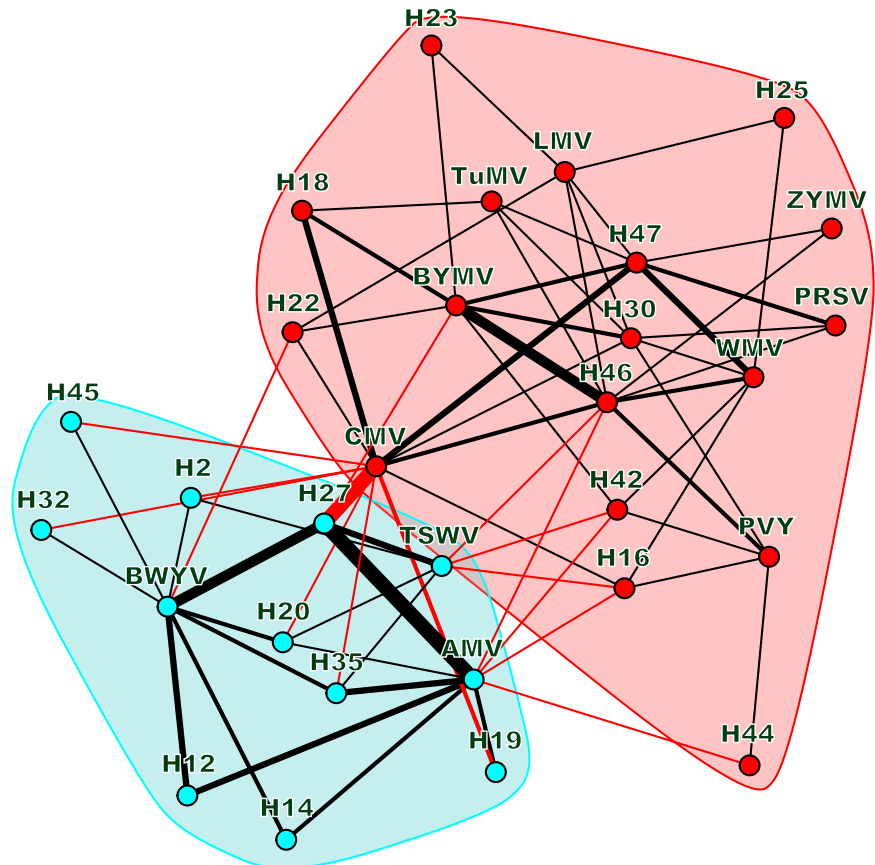

Fig. 3. An undirected and weighted coinfection network of Edge. The graph was produced using the random walk algorithm to detect network modules. Host species are coded $\mathrm{H}$. The color areas around nodes indicates a module. Red links indicate connections between modules, and black links define within-module connections. AMV, Alfalfa mosaic virus; BWYV, Beet Western yellow virus; BYMV, Bean yellow mosaic virus; CMV, Cucumber mosaic virus; LMV, Lettuce mosaic virus; PRSV, Papaya ringspot virus; PVY, Potato virus Y; TSWV, Tomato spotted wilt virus; TuMV, Turnip mosaic virus; WMV, Watermelon mosaic virus; and ZYMV, Zucchini yellow mosaic virus.
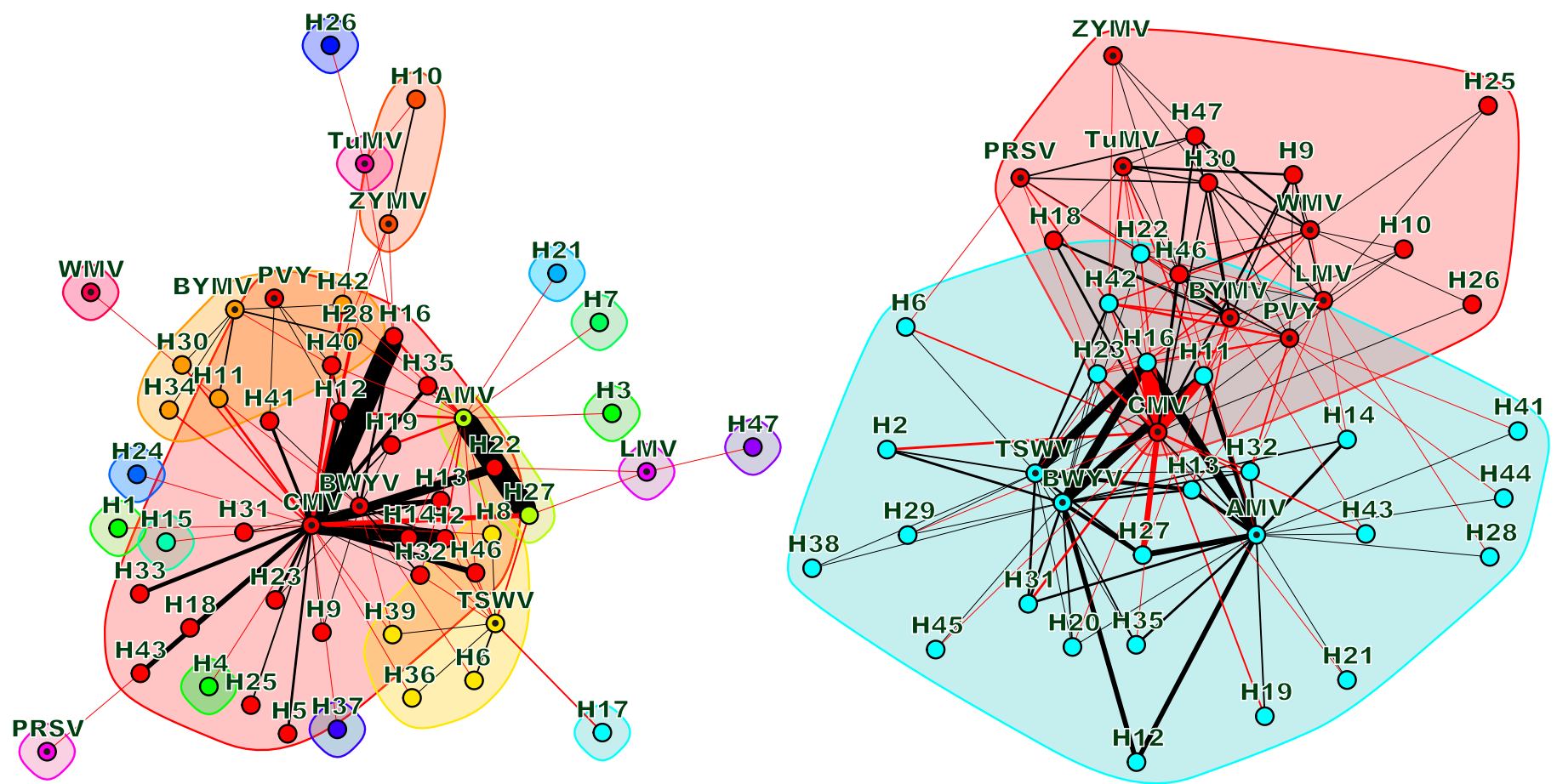

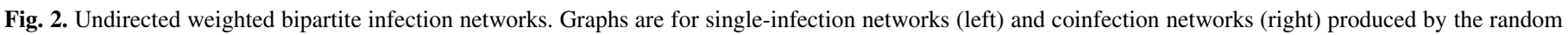

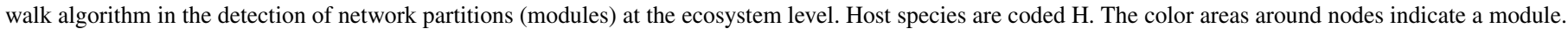

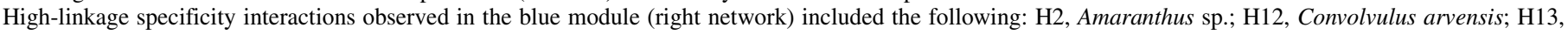

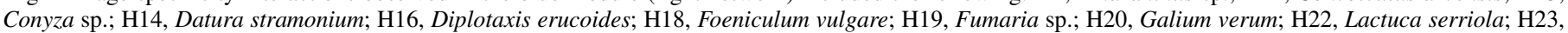

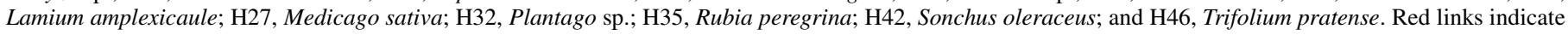

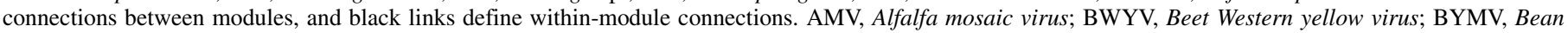

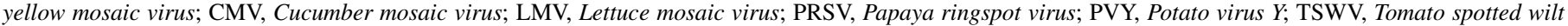
virus; TuMV, Turnip mosaic virus; WMV, Watermelon mosaic virus; and ZYMV, Zucchini yellow mosaic virus. 
resulted in weak contributions to total variance unless combined in a nested term that indicated that seasonal effects were associated with at least one of the habitats. A factor for host species had a much stronger effect on co-prevalence compared with virus species until a nested factor for either single-infection or coinfection mode was added. These strong contributions indicated cases of single infections or coinfections that were structured among individual host and virus species. The prediction of co-prevalence was improved when infection modes of both virus and host plant species were included in the model (Supplementary Table S5). These results together indicated weak contributions by seasonal and habitat variation. Host abundance was always significant and negatively related to co-prevalence, with relatively weak effects from host diversity $\left(S_{q}\right)$ that were significant only when the model included a predictor factor for host species.

We reshaped the data to address explicitly the effects of each habitat on infection heterogeneity. The contribution of coinfection level to total variance was negligible after more than three infections (Supplementary Table S6). The similarity in low AIC model fit values and high RDT scores among the coinfection model designs up to more than two infections contrasted the single-infection design with relative weak model fit. The model for more than one infection had the lowest AIC model fit and highest RDT probability. Species relative abundance in each habitat was a significant [Wald $\left.\chi^{2}{ }_{(1)}, P<0.0001\right]$ predictor of single infections (Table 1). The relationship between abundance and co-prevalence was significant [Wald $\chi^{2}(1), P<0.011$ ] in Edge habitat for up to more than two infections (Table 1), but it was nonsignificant for any level of coinfection in the other habitats. Edge had the only significant abundance effect in relation to coinfection and the prediction of co-prevalence.

\section{DISCUSSION}

This is the first study that we are aware of that investigates the structuring of individuals and species in multispecies plant-virus systems. By comparing network with regression analyses and treating coinfection separately from single-infection interactions, we found that both approaches agreed on a unique role for Edge in transmission. The network analysis showed that coinfection was the most frequent mode of infection in the ecosystem and contributed more to ecological interactions than single infections. Evidence of frequent encounters among specific viruses and hosts, multiple infections of up to six viruses in an individual, and greater than expected modularity owing to coinfection all indicated nonrandom associations. This agreed with our first main hypothesis: that coinfection contributes more than single infection to nonrandom associations and ecological interactions between viruses and hosts. The most notable finding was that the same patterns of ecological interactions among species occurred at two spatial scales (i.e., the ecological interactions of Edge and the ecosystem coinfection networks suggest that they were independent of scale). This pattern of self-similarity in modularity between networks suggests scale invariance (Wu and Li 2006). The pattern was produced only when ecological interactions in the network were defined by coinfection.

The observations from the networks clearly paralleled the significant results that emerged from the GLMMs. For instance, the significant effect of Edge habitat diversity on infection

TABLE 1. Explanatory variables of habitat-level generalized linear mixed models for co-prevalence $(n=47)^{\mathrm{a}}$

\begin{tabular}{|c|c|c|c|c|c|c|c|c|}
\hline & Estimate & SE & $\chi^{2}$ & $\mathrm{df}$ & $P\left(>\chi^{2}\right)$ & AIC & $\Delta \mathrm{AIC}$ & RDT \\
\hline One infection & & & & & & 47.4 & 9.9 & 0.759 \\
\hline (Intercept) & -0.354 & 0.008 & & & & & & \\
\hline ED abundance & -0.203 & 0.008 & 715.978 & 1 & $<2.2 \mathrm{e}-16$ & & & \\
\hline FF abundance & -0.037 & 0.008 & 23.435 & 1 & $<0.0001$ & & & \\
\hline WL abundance & -0.152 & 0.008 & 403.560 & 1 & $<2.2 \mathrm{e}-16$ & & & \\
\hline CR abundance & -0.132 & 0.008 & 304.199 & 1 & $<2.2 \mathrm{e}-16$ & & & \\
\hline More than one infection & & & & & & 37.5 & 0.0 & 0.982 \\
\hline (Intercept) & 0.097 & 0.291 & & & & & & \\
\hline ED abundance & -0.341 & 0.135 & 6.409 & 1 & 0.011 & & & \\
\hline FF abundance & -0.173 & 0.170 & 1.040 & 1 & 0.308 & & & \\
\hline WL abundance & -0.218 & 0.172 & 1.617 & 1 & 0.203 & & & \\
\hline CR abundance & -0.188 & 0.115 & 2.644 & 1 & 0.104 & & & \\
\hline More than two infections & & & & & & 38.7 & 1.2 & 0.973 \\
\hline (Intercept) & 0.161 & 0.263 & & & & & & \\
\hline ED abundance & -0.315 & 0.114 & 7.581 & 1 & 0.006 & & & \\
\hline FF abundance & -0.153 & 0.160 & 0.908 & 1 & 0.341 & & & \\
\hline WL abundance & -0.232 & 0.152 & 2.326 & 1 & 0.127 & & & \\
\hline CR abundance & -0.124 & 0.118 & 1.115 & 1 & 0.291 & & & \\
\hline More than three infections & & & & & & 45.5 & 8.0 & 0.829 \\
\hline (Intercept) & 0.069 & 0.311 & & & & & & \\
\hline ED abundance & -0.166 & 0.098 & 2.859 & 1 & 0.091 & & & \\
\hline FF abundance & -0.181 & 0.174 & 1.076 & 1 & 0.300 & & & \\
\hline WL abundance & -0.136 & 0.145 & 0.881 & 1 & 0.348 & & & \\
\hline CR abundance & -0.093 & 0.112 & 0.687 & 1 & 0.407 & & & \\
\hline More than four infections & & & & & & 48.9 & 11.4 & 0.701 \\
\hline (Intercept) & -0.331 & 0.132 & & & & & & \\
\hline ED abundance & -0.187 & 0.103 & 3.315 & 1 & 0.069 & & & \\
\hline FF abundance & -0.030 & 0.158 & 0.036 & 1 & 0.850 & & & \\
\hline WL abundance & -0.142 & 0.158 & 0.812 & 1 & 0.367 & & & \\
\hline CR abundance & -0.144 & 0.121 & 1.408 & 1 & 0.235 & & & \\
\hline More than five infections & & & & & & 48.9 & 11.4 & 0.701 \\
\hline (Intercept) & -0.331 & 0.132 & & & & & & \\
\hline ED abundance & -0.187 & 0.103 & 3.315 & 1 & 0.069 & & & \\
\hline FF abundance & -0.030 & 0.158 & 0.036 & 1 & 0.850 & & & \\
\hline WL abundance & -0.142 & 0.158 & 0.812 & 1 & 0.367 & & & \\
\hline CR abundance & -0.144 & 0.121 & 1.408 & 1 & 0.235 & & & \\
\hline
\end{tabular}

${ }^{a}$ Abundance was summed at the habitat level, with coinfection levels as random factors. The $P$ values are for the Wald $\chi^{2}$ test. AIC, Akaike information criterion; RDT, residual deviance test $P$ value; and SE, standard error. Bold text indicates significant result. ED, Edge; FF, Fallow field; WL, Wasteland; and CR, Crop. 
heterogeneity in the regression models mirrored the higher than expected modularity found only in Edge habitat from the networks. Edge was the only habitat that consistently exhibited community parameters that were significant in explaining co-prevalence. This observation supported our second main hypothesis that coinfection contributed to the distribution of infection among the habitats. However, the pattern of scale invariance and the unique properties of Edge revealed by the GLMMs suggest that the ecological interactions produced by coinfection were not wholly a function of habitat differences (i.e., compositional heterogeneity) but also, were a function of abiotic and biotic factors associated with processes wider than the extent of the ecosystem. These results together imply that ecological interactions among pivotal plant and virus species might be a stronger indicator of reservoirs of infection than single host-single pathogen interactions.

Ecological interactions and module membership. The network analyses showed that coinfection increased the number of links among particular species in the networks, which was not observable in single-infection networks. For instance, AMV, BWYV, and TSWV typically had strong interactions with hosts such as D. erucoides, M. sativa, Conyza sp., C. arvense, and Lamium amplexicaule (Fig. 3). All of the potyviruses co-occurred in the same module and constituted proportionally more coinfections among their hosts. Although these potyviruses have different host ranges and vector specificities, potyviruses can complement the transmission of each other in mixed infections (Pirone and Blanc 1996). The coinfection networks showed that CMV often coinfected with PVY, BYMV, LMV, WMV, TuMV, and PRSV in hosts that belonged to another module. Pathogens, such as CMV, with a wide ecological niche (Sacristán et al. 2004) theoretically create stable ecological associations important to the evolution of specialized resource use (Ings et al. 2009). Species that connect many others, like CMV, that may not normally interact directly have been referred to as "super-generalists" (Olesen et al. 2007). Supergeneralists can decrease the frequency of coevolutionary events, because they impose similar selective pressures on the species that they interact with (Guimarães et al. 2011). Frequently interacting species have been termed "hubs" and can refer to either individual species or members of densely linked network modules (Ings et al. 2009). Host plants, such as D. erucoides and L. serriola, that acted as hubs might have a role in maintaining trait interactions among many viruses. The wider ecological associations of "generalist" viruses might reduce specific pairwise-like trait interactions and result in relatively diffuse within-host selective mechanisms (Karasov et al. 2014). This suggests that the contribution of coinfection to increasing ecological interactions promotes multispecies coevolutionary relationships (Toju et al. 2017) and underlies evolution in the plant-pathogen system.

The pattern of scale invariance in Edge was conspicuous, because it was the only habitat that exhibited ecological interactions that corresponded to that of the ecosystem. We removed all nodes and links of Edge from an ecosystem-level network analysis $\left[t_{(999)}=\right.$ $137.57, P<0.0001]$ to positively confirm (two modules, one with AMV, BWYV, and TSWV) that the scale invariance at the larger scale was independent of Edge interactions. Although there was a significant $\left[\chi_{(3)}^{2}=23.41, P<0.0001\right]$ habitat effect on the proportions of single infections and coinfections, no clear pattern emerged from the networks that could explain the distribution of the infection modes among habitats. For instance, the number of infections (links) of each infection mode in each habitat did not significantly $\left[\chi^{2}{ }_{(7)}=0.134, P=1.000\right]$ correspond with connectance ( $C$; another type of measurement of ecological interactions). Virus host range had a role in infection heterogeneity (Fig. 1) among hosts and correlated with more infections $\left(r^{2}=0.954, P<0.0001\right)$ in general. However, host range did not seem to relate directly to ecological interactions, because it did not correspond to frequencies of either single infection or coinfection. For instance, in Crop, CMV with the widest host range had strong single-infection interactions with Amaranthus sp. but no coinfections. The reverse scenario was observed in Edge, where CMV coinfections dominated the interactions with Amaranthus sp. Similarly, AMV had strong singleinfection interactions with $M$. sativa but far fewer coinfections in Edge. Ecological interactions were a function of coinfection and the composition and identity of host species present in Edge. Together, this suggests that ecological interactions and transmission among particular pathogens and hosts are contingent on spatial dependencies.

Habitat-independent species interactions and patterns consistent with scale invariance both suggest that transmission depended on ecological interactions (i.e., coinfection) and the spatial organization of virus and host species resources. Virus infection and host susceptibility traits may, therefore, be disconnected from one another at a given space and time. For instance, the widest host ranges and highest total number of infections were not positively correlated with our measure of infection heterogeneity (Supplementary Fig. S10). Furthermore, patterns produced by the networks suggest that the viruses with the widest host ranges had distinct ecological interactions that varied among the habitats. Shared module membership among the potyviruses, the unique role that CMV had in connecting communities, and conserved module membership of taxonomically divergent viruses together implied higher-level organization of epidemiological processes. Species membership distinctions between modules might be analogous to between-community ecological niche differentiation ( $\beta$-niche). In other studies, $\beta$-diversity variation in plant-virus interactions was correlated with climate and host abundance (Biddle et al. 2012; Fraile et al. 2017). Modules may represent the $\alpha$-niche, where fitness is largely dependent on within-community coexistence (Devictor et al. 2010). Because environmental change may alter contact rates within or between communities, the fitness landscape under which virus-plant or virus-virus evolution occurs may also change (Estrada-Peña et al. 2014). Selection for wider or narrower resource use strategies and transmission dynamics will, therefore, depend on the difference between the rate of ecological niche change and the rate at which a population can adapt to it (Elena and Sanjuán 2003). Additionally, scale invariance of ecological interactions implies that the ecological niches that constrain or promote pathogen evolution and emergence are also spatially variable, because resource dimensions that define a niche are subject to spatial dependencies. Consequently, different infectivity or resistance phenotypes might respond to processes that propagate at different scales. Additionally, the scale of a process may also vary according to the spatial and temporal arrangements of species and other resources.

Ecological interactions, coinfection, and infection heterogeneity. It must be emphasized that the strength of ecological network analyses is in their ability to reveal focal species and community dynamics, but they are limited in their ability to link these patterns to causal mechanisms. However, the GLMM analyses provided a contrasting approach that enabled the evaluation of specific community parameters in addition to the effects of coinfection on structuring infection. The GLMMs showed that compositional heterogeneity (differences in host abundances among the habitats) best explained infection heterogeneity only under single infections (Table 1), because there was a significant effect by all habitats under this model design. When coinfection was specified in the models, Edge produced the only significant relationship between community parameters and infection heterogeneity for up to more than three infections (Table 1). Infection heterogeneity depended on particular interactions among certain species (i.e., hubs) and not just the number of links (i.e., infections). For example, Wasteland had lower relative abundances and higher co-prevalence than Edge (Supplementary Fig. S11), but it also had lower than expected modularity. The viruses that contributed to most single infections and coinfections (and ecological interactions) overall (AMV, 
BWYV, CMV, and TSWV) were associated with high abundance hosts and not generally associated with high co-prevalence. This suggests that predicting ecological processes that cause infection heterogeneity and transmission dynamics partly relies on identifying scale dependencies among hub species.

The results of GLMMs (Table 1) together with patterns produced by the network analyses suggest a connection between the community parameters of Edge and the higher than expected network modularity of Edge. As we have seen from discrepancies between the structural patterns observed among hosts and viruses in Edge and the other habitats, ecological processes that correspond to infection heterogeneity and transmission might not have to correspond to the same scale (i.e., exhibit scale invariance). Biodiversity can influence infection prevalence in different ways (Strauss et al. 2015), and this relationship changes according to the spatial scale and grain size at which it is measured (McLeish et al. 2017). For example, vector preferences for plant species and preference variability among communities influence the spatial structuring of coinfection at large (Power et al. 2011) and small (within-host) scales (Susi et al. 2015). One possibility explaining the different properties of Edge is the relationship between virus prevalence and plant biomass uncovered by Sacristán et al. (2004). The biomass in crop boundaries throughout the year was more constant than in Wasteland habitat. This suggested the higher plant biomass supported higher vector populations and increased transmission. Uncovering the epidemiology of multihost pathogens is a major hurdle in identifying reservoirs (Viana et al. 2014). Our results reveal the importance of Edge as a reservoir community (Ashford 2003) that included both plant and virus transmission hubs, which might intensify ecological interactions and coprevalence in close proximity to crops. Epidemiological network analysis has shown that increased ecological interactions can lead to lower thresholds for epidemic outbreaks (Yang et al. 2015). Transmission to crop from reservoirs and vice versa will depend on their ecological interactions (e.g., Gurarie and Seto 2008) and the compositional features of the ecosystem. This suggests that the spatial organization of transmission hubs that result in a reservoir might create stable ecological niches or modify evolutionary dynamics that inhibit or promote emergence in crops.

Conclusion-what drives transmission? It was expected that coinfection would increase the complexity of ecological interactions and virus transmission owing to variability in (i) the number of infections within hosts and (ii) the frequencies of infections between hosts. To the contrary, we found that coinfection might be associated with stable ecological interactions, especially between key host and virus species that we termed hubs. Transmission hubs that connect communities of hosts and viruses possibly drive diffuse multispecies coevolutionary dynamics. The implications of both network and regression modeling point to community-level organization that indicates reservoirs of relatively stable ecological interactions. These findings showed that key species as well as communities of viruses and hosts provide useful information about components of transmission at the ecosystem level. Identifying hubs of transmission and potential reservoirs of infection is a powerful device for selective management strategies in disease control and an important step in determining whether stable ecological networks either promote or inhibit pathogen emergence. If stable ecological associations are common in agroecosystems, then anticipating or predicting transmission pathways will be less uncertain. Our findings raised at least two possibilities: that (i) Edge reservoir communities drove ecological interactions and infection heterogeneity of the entire ecosystem (Nishiura et al. 2009) or that (ii) Edge communities supported sufficient transmission hubs to reproduce a global pattern. Or, is it a confluence of processes propagating from multiple scales? In other words, which way does the signal, or effect, propagate? These hypotheses leave an interesting line of investigation for future research.

\section{LITERATURE CITED}

Ashford, R. W. 2003. When is a reservoir not a reservoir? Emerg. Infect. Dis. 9:1495-1496.

Barabási, A. L., and Albert, R. 1999. Emergence of scaling in random networks. Science 286:509-512.

Barrett, L. G., Kniskern, J. M., Bodenhausen, N., Zhang, W., and Bergelson, J. 2009. Continua of specificity and virulence in plant host-pathogen interactions: Causes and consequences. New Phytol. 183:513-529.

Bates, D., Maechler, M., Bolker, B., and Walker, S. 2015. Fitting linear mixedeffects models using lme4. J. Stat. Softw. 67:1-48.

Biddle, J. M., Linde, C., and Godfree, R. C. 2012. Co-infection patterns and geographic distribution of a complex pathosystem targeted by pathogenresistant plants. Ecol. Appl. 22:35-52.

Buhnerkempe, M. G., Roberts, M. G., Dobson, A. P., Heesterbeek, H., Hudson, P. J., and Lloyd-Smith, J. O. 2015. Eight challenges in modelling disease ecology in multi-host, multi-agent systems. Epidemics 10:26-30.

Clark, M. F., and Adams, A. N. 1977. Characteristics of the microplate method of enzyme-linked immunosorbent assay for the detection of plant viruses. J. Gen. Virol. 34:475-483.

Craft, M. E., Volz, E., Packer, C., and Meyers, L. A. 2010. Disease transmission in territorial populations: The small-world network of Serengeti lions. J. R. Soc. Interface 8:776-786.

Csardi, G., and Nepusz, T. 2006. The igraph software package for complex network research. InterJournal. Complex Syst. 1695:1-9.

Devictor, V., Clavel, J., Julliard, R., Lavergne, S., Mouillot, D., Thuiller, W., Venail, P., Villeger, S., and Mouquet, N. 2010. Defining and measuring ecological specialization. J. Appl. Ecol. 47:15-25.

Elena, S. F., and Sanjuán, R. 2003. Climb every mountain? Science 302: 2074-2075.

Estrada-Peña, A., Ostfeld, R. S., Peterson, A. T., Poulin, R., and de la Fuente, J. 2014. Effects of environmental change on zoonotic disease risk: An ecological primer. Trends Parasitol. 30:205-214.

Fahrig, L., Baudry, J., Brotons, L., Burel, F. G., Crist, T. O., Fuller, R. J., Sirami, C., Siriwardena, G. M., and Martin, J. L. 2011. Functional landscape heterogeneity and animal biodiversity in agricultural landscapes. Ecol. Lett. 14:101-112.

Fraile, A., McLeish, M. J., Pagán, I., González-Jara, P., Piñero, D., and García-Arenal, F. 2017. Environmental heterogeneity and the evolution of plant-virus interactions: Viruses in wild pepper populations. Virus Res. 241:68-76.

Guimarães, P. R., Jr., Jordano, P., and Thompson, J. N. 2011. Evolution and coevolution in mutualistic networks. Ecol. Lett. 14:877-885.

Gurarie, D., and Seto, E. Y. 2008. Connectivity sustains disease transmission in environments with low potential for endemicity: Modelling schistosomiasis with hydrologic and social connectivities. J. R. Soc. Interface 6: 495-508.

Ings, T. C., Montoya, J. M., Bascompte, J., Blüthgen, N., Brown, L., Dormann, C. F., Edwards, F., Figueroa, D., Jacob, U., Jones, J. I., and Lauridsen, R. B. 2009. Ecological networks-beyond food webs. J. Anim. Ecol. 78:253-269.

Karasov, T. L., Kniskern, J. M., Gao, L., DeYoung, B. J., Ding, J., Dubiella, U., Lastra, R. O., Nallu, S., Roux, F., Innes, R. W., and Barrett, L. G. 2014. The long-term maintenance of a resistance polymorphism through diffuse interactions. Nature 512:436-440.

Kones, J. K., Soetaert, K., van Oevelen, D., and Owino, J. 2009. Are network indices robust indicators of food web functioning? A Monte Carlo approach. Ecol. Modell. 220:370-382.

Krause, A. E., Frank, K. A., Mason, D. M., Ulanowicz, R. E., and Taylor, W. W. 2003. Compartments revealed in food-web structure. Nature 426:282-285.

Lello, J., Boag, B., Fenton, A., Stevenson, I. R., and Hudson, P. J. 2004. Competition and mutualism among the gut helminths of a mammalian host. Nature 428:840-844.

Leventhal, G. E., Hill, A. L., Nowak, M. A., and Bonhoeffer, S. 2015. Evolution and emergence of infectious diseases in theoretical and real-world networks. Nat. Commun. 6.

Lewinsohn, T. M., Inácio Prado, P., Jordano, P., Bascompte, J., and Olesen, J. M. 2006. Structure in plant-animal interaction assemblages. Oikos 113: 174-184.

Malmstrom, C. M., Stoner, C. J., Brandenburg, S., and Newton, L. A. 2006. Virus infection and grazing exert counteracting influences on survivorship of native bunchgrass seedlings competing with invasive exotics. J. Ecol. 94: 264-275.

Malpica, J. M., Sacristán, S., Fraile, A., and García-Arenal, F. 2006. Association and host selectivity in multi-host pathogens. PLoS One 1:e41.

McLeish, M., Sacristán, S., Fraile, A., and García-Arenal, F. 2017. Scale dependencies and generalism in host use shape virus prevalence. Proc. R. Soc. Lond. B Biol. Sci. 284:20172066.

Newman, M. E., and Girvan, M. 2004. Finding and evaluating community structure in networks. Phys. Rev. E 69:026113. 
Nishiura, H., Hoye, B., Klaassen, M., Bauer, S., and Heesterbeek, H. 2009. How to find natural reservoir hosts from endemic prevalence in a multi-host population: A case study of influenza in waterfowl. Epidemics 1:118-128.

Olesen, J. M., Bascompte, J., Dupont, Y. L., and Jordano, P. 2007. The modularity of pollination networks. Proc. Natl. Acad. Sci. USA 104:19891-19896.

Ostfeld, R. S., and Keesing, F. 2000. The function of biodiversity in the ecology of vector-borne zoonotic diseases. Can. J. Zool. 78:2061-2078.

Perkins, T. A., Scott, T. W., Le Menach, A., and Smith, D. L. 2013. Heterogeneity, mixing, and the spatial scales of mosquito-borne pathogen transmission. PLoS Comput. Biol. 9:e1003327.

Pirone, T. P., and Blanc, S. 1996. Helper-dependent vector transmission of plant viruses. Annu. Rev. Phytopathol. 34:227-247.

Pons, P., and Latapy, M. 2006. Computing communities in large networks using random walks. Pages 284-293 in: Proceedings of the International Symposium on Computer and Information Sciences, Yolum, T. Güngör, F. Gürgen, and C. Özturan, eds. Springer, Berlin.

Power, A. G., Borer, E. T., Hosseini, P., Mitchell, C. E., and Seabloom, E. W. 2011. The community ecology of barley/cereal yellow dwarf viruses in Western US grasslands. Virus Res. 159:95-100.

Power, A. G., and Mitchell, C. E. 2004. Pathogen spillover in disease epidemics. Am. Nat. 164:S79-S89.

R Core Team. 2012. R: A Language and Environment for Statistical Computing. R Foundation for Statistical Computing, Vienna, Austria.

Rottstock, T., Joshi, J., Kummer, V., and Fischer, M. 2014. Higher plant diversity promotes higher diversity of fungal pathogens, while it decreases pathogen infection per plant. Ecology 95:1907-1917.

Sacristán, S., Fraile, A., and García-Arenal, F. 2004. Population dynamics of Cucumber mosaic virus in melon crops and in weeds in central Spain. Phytopathology 94:992-998.

Salvaudon, L., De Moraes, C. M., and Mescher, M. C. 2013. Outcomes of coinfection by two potyviruses: Implications for the evolution of manipulative strategies. Proc. R. Soc. Lond. B Biol. Sci. 280:20122959.

Seabloom, E. W., Borer, E. T., Mitchell, C. E., and Power, A. G. 2010. Viral diversity and prevalence gradients in North American Pacific Coast grasslands. Ecology 91:721-732.

Seabloom, E. W., Hosseini, P. R., Power, A. G., and Borer, E. T. 2009. Diversity and composition of viral communities: Coinfection of barley and cereal yellow dwarf viruses in California grasslands. Am. Nat. 173: E79-E98.

Strauss, A. T., Civitello, D. J., Cáceres, C. E., and Hall, S. R. 2015. Success, failure and ambiguity of the dilution effect among competitors. Ecol. Lett. 18:916-926.

Streicker, D. G., Fenton, A., and Pedersen, A. B. 2013. Differential sources of host species heterogeneity influence the transmission and control of multihost parasites. Ecol. Lett. 16:975-984.

Susi, H., Vale, P. F., and Laine, A. L. 2015. Host genotype and coinfection modify the relationship of within and between host transmission. Am. Nat. 186:252-263.

Syller, J. 2012. Facilitative and antagonistic interactions between plant viruses in mixed infections. Mol. Plant Pathol. 13:204-216.

Telfer, S., Lambin, X., Birtles, R., Beldomenico, P., Burthe, S., Paterson, S., and Begon, M. 2010. Species interactions in a parasite community drive infection risk in a wildlife population. Science 330:243-246.

Toju, H., Yamamichi, M., Guimarães, P. R., Jr., Olesen, J. M., Mougi, A., Yoshida, T., and Thompson, J. N. 2017. Species-rich networks and ecoevolutionary synthesis at the metacommunity level. Nat. Ecol. Evol. 1:0024.

Tollenaere, C., Susi, H., and Laine, A. L. 2016. Evolutionary and epidemiological implications of multiple infection in plants. Trends Plant Sci. 21: 80-90.

Viana, M., Mancy, R., Biek, R., Cleaveland, S., Cross, P. C., Lloyd-Smith, J. O., and Haydon, D. T. 2014. Assembling evidence for identifying reservoirs of infection. Trends Ecol. Evol. 29:270-279.

Whitfield, A. E., Falk, B. W., and Rotenberg, D. 2015. Insect vector-mediated transmission of plant viruses. Virology 479:278-289.

Woolhouse, M. E., Taylor, L. H., and Haydon, D. T. 2001. Population biology of multihost pathogens. Science 292:1109-1112.

Woolhouse, M. E., Webster, J. P., Domingo, E., Charlesworth, B., and Levin, B. R. 2002. Biological and biomedical implications of the co-evolution of pathogens and their hosts. Nat. Genet. 32:569-577.

Wu, J., and Li, H. 2006. Concepts of scale and scaling. Pages 3-15 in: Scaling and Uncertainty Analysis in Ecology, J. Wuk, B. Jonesharbin, and L. L. Loucks, eds. Springer, Dordrecht, The Netherlands.

Yang, H., Tang, M., and Gross, T. 2015. Large epidemic thresholds emerge in heterogeneous networks of heterogeneous nodes. Sci. Rep. 5:13122. 